\title{
Optical communication based on the periodic nonlinear Fourier transform signal processing
}

\author{
Morteza Kamalian Kopae, Jaroslaw E. Prilepsky, Son Thai Le, and Sergei K. Turitsyn \\ Aston Institute of Photonic Technologies, Aston University, Birmingham, B4 7ET, UK \\ Email: kamaliam@aston.ac.uk
}

\begin{abstract}
In this work we introduce the periodic nonlinear Fourier transform (PNFT) and propose a proof-of-concept communication system based on it by using a simple waveform with known nonlinear spectrum (NS). We study the performance (addressing the bit-error-rate (BER), as a function of the propagation distance) of the transmission system based on the use of the PNFT processing method and show the benefits of the latter approach. By analysing our simulation results for the system with lumped amplification, we demonstrate the decent potential of the new processing method.
\end{abstract}

\section{INTRODUCTION}

Skyrocketing capacity demand due to the quickly emerging bandwidth-consuming on-line services (like cloud computing, HD on-demand video streams, etc.) and corresponding increasing requirements for the data rates in the fiber-optic communication systems stimulate the continuous progress in optical communication technology [1], [2]. Inverse scattering transform method, also known as the nonlinear Fourier transform (NFT), is a well-developed tool for solving the socalled integrable nonlinear equations [2], [3]. The nonlinear Schrödinger equation (NLSE), which is a common model describing the electrical field evolution along a single-mode optical fiber, belongs to the aforementioned class of equations that can be solved by the NFT. Various modifications of the NFT-based approaches have been recently intensively studied as an efficacious tool for the nonlinearity compensation in high spectral efficiency (SE) optical transmission systems [2], [4][14]. The original idea of using the NFT and the associated NS for the data transmission dates back to the pioneering work on eigenvalue communications by Hasegawa and Nyu [15] from 1993. The advantage of the NFT-based fiber-optic communications is that the nonlinearity is included into the NFT processing, and, in turn, the NS propagation inside the NFT domain is essentially linear [2]. Thus, the nonlinearity itself becomes an undetachable part of the processing method.

Within the NFT-based approach we can define the analog of Fourier spectrum - the NS associated with a given input waveform $q(t, 0)$ in the time domain (at the initial distance $z=0$ ). Then we propagate the NS towards the receiver. If one seeks for the resulting waveform $q(t, L)$ at the end of the fiber, say at $z=L$, we can go back to the temporal domain by employing the inverse transform (INFT) using the evolved nonlinear spectrum. At this point, we note that all previous studies with regard to the NFT-based transmission methods exploited the infinite-line (ordinary) version of the
NFT, where it is explicitly assumed that the signal decays (at least, exponentially) when $t \rightarrow \pm \infty$. This fact caused some processing difficulties and performance degradation, as one needs to keep the whole signal inside the processing (symbol duration) window, while some parts of the evolved signal can already escape out of this window at the receiver causing the inter-symbol interference (ISI). For the nonlinear inverse synthesis method [7]-[9], the INFT-based signal synthesis results in the effective broadening of the generated waveshape in time domain, thus reducing the overall SE of the communication system, and the latter effect becomes a serious limitation at high signal powers where the NFT usage could become most beneficial. On the other hand, the processing based on the PNFT can bring the following benefits. One of the challenges of any communication systems is the signal broadening due to the chromatic dispersion (CD). The CD effects can be compensated either at the transmitter, along the fiber or at the receiver [1]. In an ordinary fiber link, the usage of filters to compensate the $\mathrm{CD}$ introduces delay and additional costs. Another way of dealing with this problem is to insert guard intervals (exceeding the channel memory) to prevent the ISI or to use the burst mode at the expense of lowering the effective data rates [8]. Using the periodic signals equipped with cyclic extensions leads to the ISI mitigation while one can still keep the processing window as small as the data-bearing part. As shown in Fig. 1, to recover the signal in the burst mode (upper part of the figure), which is the usual requirement for the traditional NFT-based methods [8], [9], [11], one has to gather all signal-related samples inside an extended window according to the channel memory, as opposed to the continuous mode (lower part of the figure), in which all the information stays within the data-bearing part of the received signal. This reduces the computational burden especially in high data rate communication where the channel memory is large. Moreover, one of the difficulties of ordinary NFT-based communication systems is that the set of signals from which the transmitting one is drawn, basically comprises elements with possibly different signal characteristics such as bandwidth and time duration [11]. This is, however, not an issue for the PNFT inasmuch as in this case the time duration of signal (period) is fixed for all participating signals (symbols) and one can fix a limited variation of the bandwidth for all symbols. Along with this, one can mention the simpler signal synthesis and the absence of power gaps in-between the bursts in the stream as additional advantages of the PNFT over the 


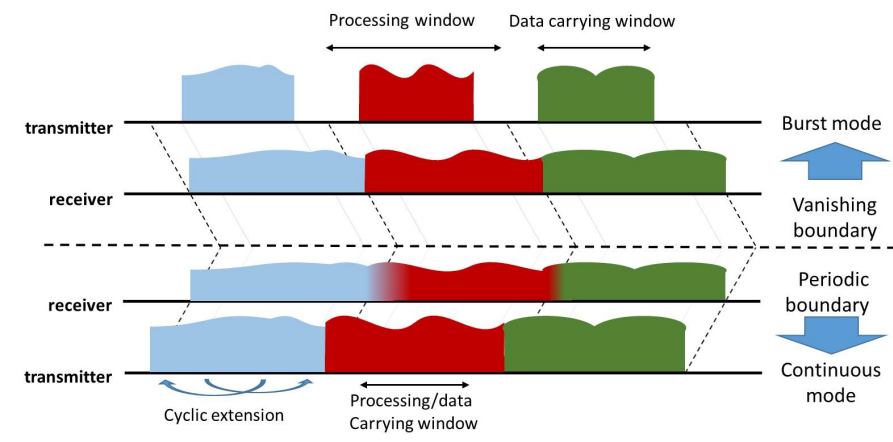

Fig. 1: Comparing the processing window necessary to mitigate the ISI at the receiver in burst mode (upper part) and continuous mode (lower part).

ordinary NFT-based processing.

\section{BASICS OF DIRECT PNFT}

PNFT is based on the calculation of the so-called monodromy matrix obtained from the Zakharov-Shabat system (ZSS). ZSS associated to the NLSE was introduced in [3]. The result of the transformation is the NS which consists of the main and auxiliary parts. The process of finding the NS from the signal is the direct PNFT. Genrally, the NS comprises the dynamical and conserved quantities (the latter, i.e. the conserved eigenvalues were utilized in the eigenvalue communication idea [15]). For the PNFT the conserved quantities constitute the so-called main spectrum, and in this work we deal with this conserved spectrum part.

The NLSE describing the signal propagation is written as

$$
j q_{z}-\frac{\beta_{2}}{2} q_{t t}+\gamma q|q|^{2}=0,
$$

where $q(t, z)$ is the slowly varying envelop of the electromagnetic field, $\beta_{2}<0$ is a chromatic dispersion parameter and $\gamma$ is a nonlinearity (Kerr) coefficient. After the standard normalizations [8], [9], we arrive at the NLSE in the form $j q_{z}+q_{t t}+2 q|q|^{2}=0$ for a periodic signal $q(t, z)$, with the period $T: q(t, z)=q(t+T, z)$. We aim at defining the NS using the associated periodic version of the ZSS [3]:

$$
\left[\begin{array}{cc}
i \partial_{t} & q(t, z) \\
-q^{*}(t, z) & -i \partial_{t}
\end{array}\right]\left[\begin{array}{l}
\phi_{1} \\
\phi_{2}
\end{array}\right]=\lambda\left[\begin{array}{l}
\phi_{1} \\
\phi_{2}
\end{array}\right],
$$

and find the eigenvalues, $\lambda$, and the eigenfunctions $\Phi=$ $\left[\phi_{1}, \phi_{2}\right]^{T}$, to form the fundamental matrix comprising two linearly independent ZSS solutions with the conditions set at some point $t_{0}[16]$ :

$$
\phi\left(t_{0}, t_{0} ; \lambda\right)=\left(\begin{array}{l}
1 \\
0
\end{array}\right), \quad \tilde{\phi}\left(t_{0}, t_{0} ; \lambda\right)=\left(\begin{array}{l}
0 \\
1
\end{array}\right) .
$$

The monodromy matrix $M$ is then defined as the value of the fundamental matrix at one period from the base point [16]: $M=\left[\phi\left(t_{0}+T, t_{0} ; \lambda\right), \tilde{\phi}\left(t_{0}+T, t_{0} ; \lambda\right)\right]^{T}$. The main spectrum is defined as points, $\lambda_{i}$, in the complex plane at which the ZSS solutions are anti-periodic or periodic:

$$
\Gamma=\left\{\lambda_{i} ; M_{11}\left(t_{0}, \lambda_{i}\right)+M_{22}\left(t_{0}, \lambda_{i}\right)= \pm 2\right\} .
$$

Main spectrum remains invariant along the signal transmission in the unperturbed NLSE. This property gives as an opportunity to use this main spectrum for transmitting our encoded data uncorrupted along the nonlinear fiber (the same idea but for the non-periodic NFT was proposed in [15]).

There exist several efficient numerical methods to calculate the NS associated with the PNFT of a signal, e.g. a piece-wise constant approximation, spectral collocation and AblowitzLadik discretization scheme [17]. We compared these methods (results are not provided here) and conclued that the AblowitzLadik scheme provides the best accuracy of the resulting NS. Importantly, the latter method can be recast into the superfast form using the FFT-based polynomial arithmetic [17].

\section{AN EXAMPLE OF COMMUNICATION SYSTEM BASED ON THE PNFT PROCESSING}

Taking advantage of the invariance of main spectrum, we can construct the signal corresponding to an encoded complex set of points (taken from arbitrary constellation) considering these constellation points as a main spectrum $\lambda_{i}$ of an unknown waveform in the time domain, then synthesize this waveform by the inverse PNFT, launch our generated signal into the fiber, and performing the PNFT at the receiver we retrieve the transmitted data from the set $\lambda_{i}$ (Fig. 2). Instead of carrying out the inverse PNFT to construct the signal, we use a simple example signal where the analytical expression for the main spectrum is known [18]:

$$
q(t, z)=A \frac{\cosh (\phi z-i \sigma)+B \cos (\xi t-\alpha)}{\cosh \phi z+B \cos (\xi t-\alpha)} e^{i N z} .
$$

For this simple case where the main spectrum merely consists of two purely imaginary points and their complex conjugates, the parameters used in Eq. (5) are determined by one of these points and the signal period, see [18] for details. In this way, the constellation is formed by some imaginary numbers from which, according to some encoding scheme, a point is drawn. Using the selected point, one has to calculate the parameters entering Eq. (5) and send this $q(t, 0)$ to the fiber. At the receiver, getting back the set $\lambda_{i}$ by the PNFT, the decision on the transmitted value is made (Fig. 2).

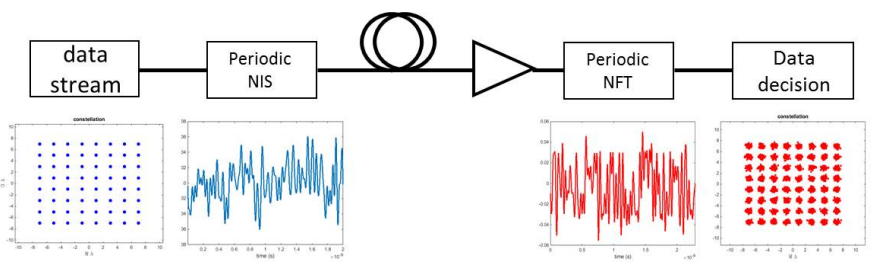

Fig. 2: Blueprint of the PNFT-based communication system. Data stream is mapped on the NS (here it is the main spectrum) and the signal in the time domain at the transmitter is constructed from this main spectrum via the inverse PNFT. At the receiver side, by performing the direct PNFT one retrieves the initial data. 


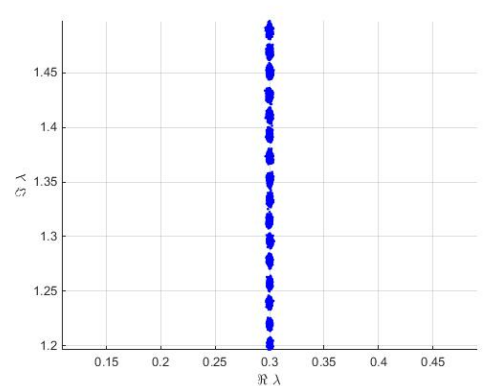

Fig. 3: Receiver constellation in a link consisting of 10 spans each having the $100 \mathrm{Km}$ length for the signal given by Eq. (5) with $2.4 \mathrm{dBm}$ power at $0.5 \mathrm{Gbaud} / \mathrm{s}(2 \mathrm{~Gb} / \mathrm{s})$ rate.

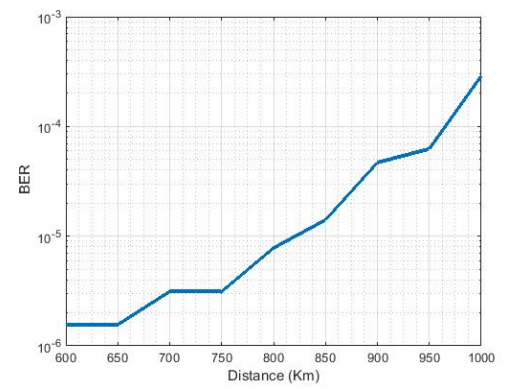

Fig. 4: BER vs propagation distance for the signal given by Eq. (5) with $2.4 \mathrm{dBm}$ power at $0.5 \mathrm{Gbaud} / \mathrm{s}(2 \mathrm{~Gb} / \mathrm{s})$.

\section{Simulation RESUlts}

For a $0.5 \mathrm{Gbaud} / \mathrm{s}$ communication, we drew a random point from a purely imaginary one-layer constellation (Fig. 3) and sent the corresponding signal into a link with 10 spans of $100 \mathrm{~km}$ length with the spontaneous emission noise added at the end of each span (each span is considered as lossless which is now plausible using Raman amplification [19]). Herein, no other linear/nonlinear distortion compensation or filtering was used. To eliminate the ISI, the signal was appended with cyclic extensions of length 3.6 times the signal length; such a significant prefix value was used due to the non-optimized characteristics in our proof-of-concept experiment. The signal power is $2.4 \mathrm{dBm}$ and we transmit signal in bundles of 16 symbols. Fig. 3 shows the received constellation at $10^{3} \mathrm{Km}$, which is degraded due to the PNFT numerical errors and (mainly) noise action. We repeated the transmission $10^{5}$ times and counted the mismatches between the transmitted bit stream and the estimated one at the receiver, which is depicted in Fig. 4 and shows that the BER is small enough for this example PNFT-based system to be counted as a reliable system.

\section{CONCLUSION AND DisCUSSION}

In this work, for the first time, we introduced the basic concepts of PNFT for fiber optical communication systems and proposed a proof-of-concept example, where instead of performing the inverse PNFT we used a simple signal with known main spectrum [18] and mapped our data onto its main spectrum. At the receiver, by performing the PNFT we retrieved the transmitted data and calculated the BER via the direct error counting. Simulation results show an adequate performance for the system proposed. However, since the signal used is quite simple, we do not expect a significant data rate by using this type of encoding. Inverse PNFT should help to compose better signals, where, for instance, the amount of cyclic extension necessary to mitigate ISI is not as high as we have had in this work (which has confined data rate to $2 \mathrm{~Gb} / \mathrm{s}$ ), and more degrees of freedom for encoding (bits in one symbol) at the same signal power can be used.

This work was supported by the UK EPSRC Programme Grant UNLOC EP/J017582/1.

\section{REFERENCES}

[1] L. B. Du, et al., "Digital Fiber Nonlinearity Compensation: Toward 1-Tb/s transport," IEEE Signal Processing Mag. 31(2), 46-56 (2014).

[2] J. E. Prilepsky and S. K. Turitsyn, "Eigenvalue communications in nonlinear fiber channels." In Odyssey of Light in Nonlinear Optical Fibers: Theory and Applications, Ch. 18, edts. K. Porsezian and R. Ganapathy, pp. 459-490 (CRC Press, 2015).

[3] V. E. Zakharov and A. B. Shabat, "Exact theory of 2-dimensional selffocusing and one-dimensional self-modulation of waves in nonlinear media," Soviet Physics-JETP, 34(1), 62-69 (1972).

[4] M. I. Yousefi and F. R. Kschischang, "Information transmission using the nonlinear Fourier transform, Parts I-III.” IEEE Trans. Inform. Theory 60(7), 4312-4369 (2014).

[5] E. G. Turitsyna and S. K. Turitsyn, "Digital signal processing based on inverse scattering transform," Opt. Lett. 38(20), 4186-4188 (2013).

[6] J. E. Prilepsky, S. A. Derevyanko, and S. K. Turitsyn, "Nonlinear spectral management: linearization of the lossless fibre channel," Opt. Express 21(20), 24344-24367 (2013).

[7] J. E. Prilepsky, et al. "Nonlinear Inverse Synthesis and Eigenvalue Division Multiplexing in Optical Fiber Channels," Phys. Rev. Lett. 113(1), 013901 (2014).

[8] S. T. Le, J. E. Prilepsky, and S. K. Turitsyn, "Nonlinear inverse synthesis for high spectral efficiency transmission in optical fibers," Opt. Express 22(22), 26720-26741 (2014).

[9] S. T. Le, J. E. Prilepsky, and S. K. Turitsyn, "Nonlinear inverse synthesis technique for optical links with lumped amplification," Opt. Express, 23(7), 8317-8328 (2015).

[10] H. Bülow, "Experimental Demonstration of Optical Signal Detection Using Nonlinear Fourier Transform,' J. Lightwave Technol. 33(7), 14331439 (2015).

[11] S. Hari, F. Kschischang, and M. Yousefi, "Multi-eigenvalue communication via the nonlinear Fourier transform," 27th Biennial Symposium on Communications (QBSC), pp. 92-95, 2014.

[12] Z. Dong, et al. "Nonlinear Frequency Division Multiplexed Transmissions based on NFT," IEEE Photon. Tech. Lett. 27(15), 1621-1623 (2015).

[13] A. Maruta, "Eigenvalue Modulated Optical Transmission System (invited)," The 20th OECC, Shanghai, China, Paper JThA.21 (2015).

[14] S. T. Le, J. E. Prilepsky, M. Kamalian, P. Rosa, M. Tan, J. D. AniaCastan, Paul Harper and Sergei K. Turitsyn, "Modified Nonlinear Inverse Synthesis for Optical Links with Distributed Raman Amplification," ECOC, Valencia, Spain, paper Tu1.1.3, (2015)

[15] A. Hasegawa and T. Nyu, "Eigenvalue Communication," J. Lightwave Technol. 11(3), 395-399 (1993).

[16] E. R. Tracy and H. H. Chen, "Nonlinear self-modulation: An exactly solvable model," Phys. Rev. A, 37(3), (1988).

[17] S. Wahls and H. V. Poor, "Fast Numerical Nonlinear Fourier Transforms," arXiv:1402.1605 (2014).

[18] M. Bertola and P. Giavedoni, "A degeneration of two-phase solutions of the focusing nonlinear Schrödinger equation via Riemann-Hilbert problems," J. Math. Phys. 56(6), 061507 (2015).

[19] M. Tan, et al. "Long-haul Transmission Performance Evaluation of Ultra-long Raman Fibre Laser Based Amplification Influenced by Second Order Co-pumping," Asia Communications and Photonics Conference, China (2014). 